\title{
A differential mechanical profilometer for thickness measurement
}

\author{
J. Maia Alves, ${ }^{\text {a) }}$ M. C. Brito, J. M. Serra, and A. M. Vallêra \\ Departamento de Física, Faculdade de Ciências da Universidade de Lisboa, Campo Grande, 1700 Lisbon, \\ Portugal and Centro de Física da Matéria Condensada da Universidade de Lisboa, Lisbon, Portugal
}

(Received 21 January 2004; accepted 20 September 2004; published 2 December 2004)

\begin{abstract}
A low cost differential profilometer based on standard commercial displacement transducers is fully described. Unlike most common profilometers this device can be used to measure the thickness profile of samples having both surfaces irregular. A sensitivity of about $0.2 \mu \mathrm{m}$, independent of the sample thickness is achieved. () 2004 American Institute of Physics. [DOI: 10.1063/1.1821627]
\end{abstract}

Common mechanical profilometry is not a good technique for thickness measurement unless one of the sample surfaces is fairly flat and thus can act as a reference plane. ${ }^{1}$ If this is not the case, as usually happens with silicon ribbons for solar applications, the measurement of a thickness profile is virtually impossible to make. Alternative techniques, such as interferometry, ellipsometry, or laser-ultrasound spectroscopy, are best suited for thickness measurement of thin films, and often require the use of expensive equipment. ${ }^{2-9}$

We describe a simple and low cost differential mechanical profilometer with a resolution of about $0.2 \mu \mathrm{m}$ that can be used to overcome this difficulty. The system uses two Sangamo NER/2.5 mm/STA displacement transducers with a sensitivity close to $80 \mathrm{mV} \mathrm{V}^{-1} \mathrm{~mm}^{-1}$. Both transducers are fixed to a U-shaped aluminum support as shown in Fig. 1. The magnitude of the force applied by transducer's tips on the sample can simply be adjusted by locking both transducers in the support, more or less closer to each other.

As shown in Fig. 2, the output signals ( $X 1$ and $X 2)$ from both transducers are electronically added and this sum is then amplified. Variable resistor $R_{8}$ is adjusted to correct for different sensitivities of the transducers so that the thickness measurement is independent of the sample position relative to the $\mathrm{U}$-shaped support. Variable resistor $R_{9}$ is used to set the desired sensitivity of the system. For each position of the sensors, the variable resistor $R_{7}$ (a ten turn potentiometer installed in the front panel of the unit) is used to adjust the zero before inserting the sample between the tips.

The good linearity of the system can be seen in Fig. 3 where we represent the measured thickness of different standards. In Fig. 4 we show that, after the adjustment of $R_{8}$ is made, the thickness measurement is essentially independent of the sample position within a region of about $2 \mathrm{~mm}$ around the center of the support (position $Z=0$ ). The nonlinearity of the transducers clearly shows up beyond that interval, when the sample is forced into a position corresponding to $1 \mathrm{~mm}<|Z|<1.5 \mathrm{~mm}$. If the sample is further pushed away from the center, one of the sensors reaches its maximum extension, corresponding to the vertical lines in the graph (it should be noticed that conventional profilometer operation can be achieved with our system by fixing one of tips).

Finally, in Fig. 5, we compare our thickness measurement with a profile measured by a commercial profilometer

${ }^{a)}$ Electronic mail: jma@fc.ul.pt

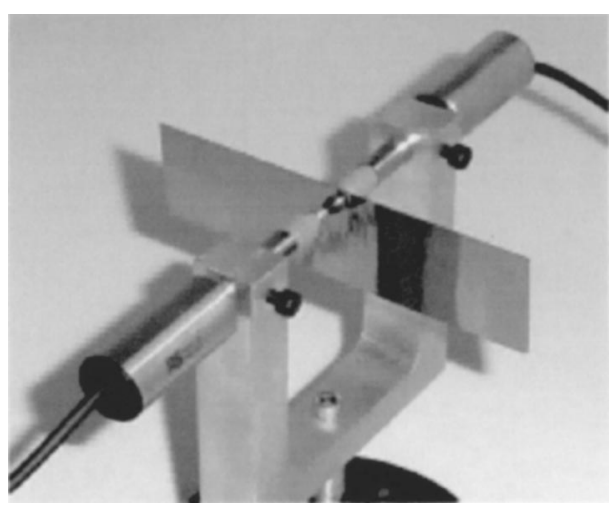

FIG. 1. View of U-shaped support with both transducers. A $250 \mu \mathrm{m}$ thick silicon ribbon is inserted between transducer's tips.

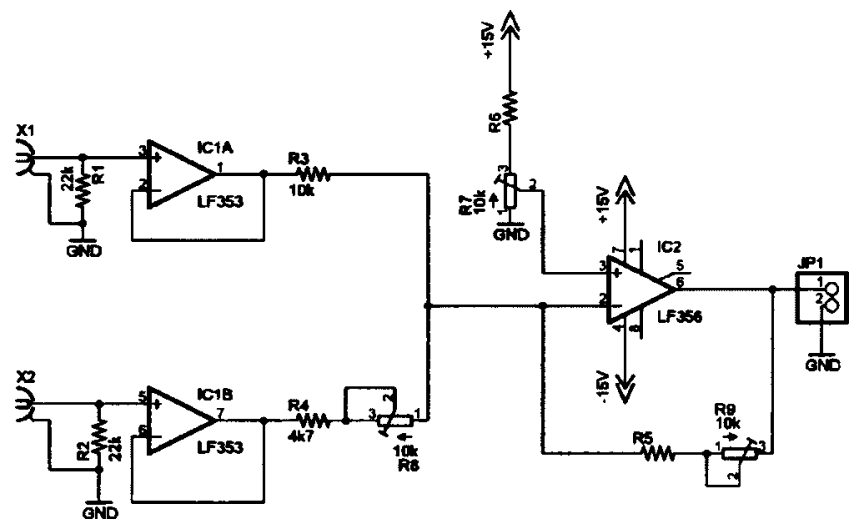

FIG. 2. Signal conditioner schematics.

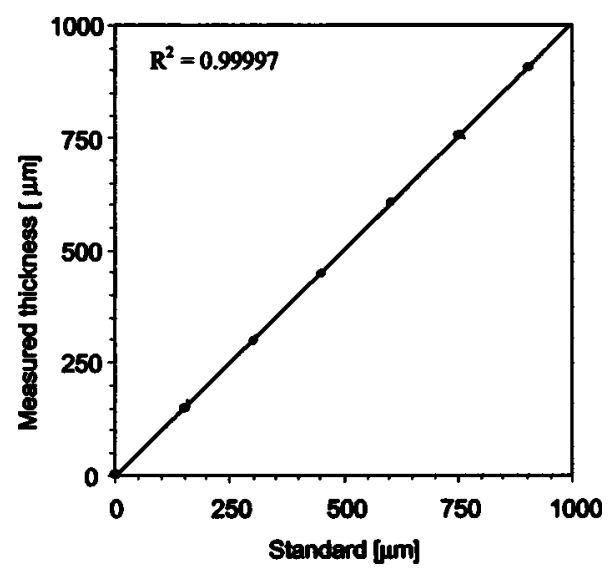

FIG. 3. Measured thickness of different standards. 


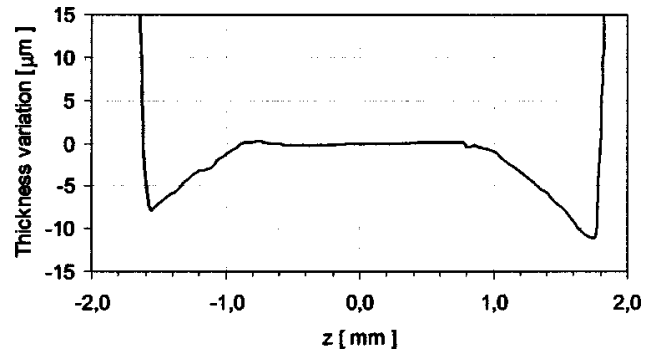

FIG. 4. Measured thickness dependence on the sample position. Position $z=0$ corresponds to the center of the U-shaped support.

(DEKTAK IIA) for a sample with one flat surface. The sample was moved using an $X Y$ translation scanning system with a maximum resolution of $25 \times 25 \mu \mathrm{m}^{2}$. The measured step height is of the order of $1 \mu \mathrm{m}$, corresponding to about $10^{-3}$ of the sample thickness. From this figure we can estimate the system resolution in thickness measurement to be about $0.2 \mu \mathrm{m}$, a limit imposed by the noise that can be seen in the flat regions of the sample. This value is close to the limit of $0.15 \mu \mathrm{m}$ in measurement repeatability that can be achieved with the transducers, as specified by the fabricant. Since this resolution is far beyond our needs, this point was not further investigated. It is our belief, however, that the resolution can still be improved simply by signal integration, for instance, by the introduction of a capacitor in parallel to the series $R_{5}, R_{9}$ (with loss of response time).

The lateral spatial resolution of the system can also be estimated from this figure by comparing the falling (or rising) edge of the step measured using our system with the one measured by the profilometer. We estimate this resolution to be about $0.15 \mathrm{~mm}$. This value is in good agreement with the

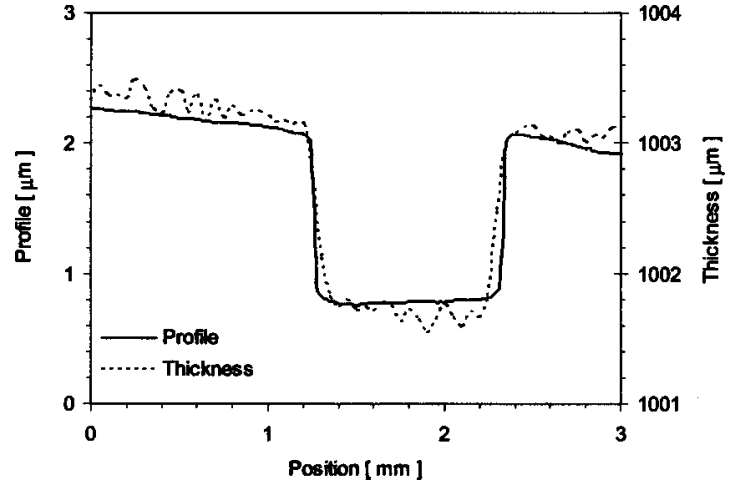

FIG. 5. Step profile comparison as measured with our system (dotted line) and with a commercial profilometer (solid line).

expected value $(0.13 \mathrm{~mm})$ considering the used tips $(3 \mathrm{~mm}$ diameter tungsten carbide ball) and the step height. Of course this can be improved, if necessary, using different tips. $^{10}$

${ }^{1}$ J. W. Wood and R. D. Redin, Rev. Sci. Instrum. 64, 2405 (1993).

${ }^{2}$ D. A. Walsh, G. U. Pukite, and J. R. Fenter, Rev. Sci. Instrum. 47, 932 (1976).

${ }^{3}$ R. J. Dewhurst, L. Noui, and Q. Shan, Rev. Sci. Instrum. 61, 1736 (1990).

${ }^{4}$ K. Hirvi, T. Mäkelä, J. Pekola, and M. Paalanen, Rev. Sci. Instrum. 65, 2735 (1994).

${ }^{5}$ G. Jin, R. Jansson, and H. Arwin, Rev. Sci. Instrum. 67, 2930 (1996).

${ }^{6}$ A. Shiratori and M. Obara, Rev. Sci. Instrum. 69, 3741 (1998).

${ }^{7}$ H. Fugimoto, Jpn. J. Appl. Phys., Part 1 38, 4256 (1999).

${ }^{8}$ A. Bardal and K. Lie, Mater. Charact. 44, 329 (2000).

${ }^{9}$ A. Moreau, D. Lévesque, M. Lord, M. Dubois, J. P. Monchalin, C. Padioleau, and J. F. Bussière, Ultrasonics 40, 1047 (2002).

${ }^{10}$ R. E. Evans, Rev. Sci. Instrum. 43, 806 (1972). 